This item was submitted to Loughborough's Research Repository by the author.

Items in Figshare are protected by copyright, with all rights reserved, unless otherwise indicated.

\title{
Generation of Secondary Solitary Waves in the Variable-Coefficient Korteweg-de Vries Equation
}

PLEASE CITE THE PUBLISHED VERSION

LICENCE

CC BY-NC-ND 4.0

\section{REPOSITORY RECORD}

Grimshaw, Roger H.J., and S.R. Pudjaprasetya. 2019. "Generation of Secondary Solitary Waves in the Variable-coefficient Korteweg-de Vries Equation”. figshare. https://hdl.handle.net/2134/302. 


\title{
Generation of Secondary Solitary Waves in the Variable-Coefficient Korteweg-de Vries Equation
}

\author{
R.H.J. Grimshaw \\ Department of Mathematical Sciences, Loughborough University, \\ Loughborough LE11 3TU, UK \\ S. R. Pudjaprasetya \\ Department of Mathematics, Institut Teknologi, Bandung, 40132, Indonesia.
}

\begin{abstract}
We consider the solitary wave solutions of a Korteweg-de Vries equation, where the coefficients in the equation vary with time over a certain region. When these coefficients vary rapidly compared with the solitary wave, then it is well-known that the solitary wave may fission into two or more solitary waves. On the other hand, when these coefficients vary slowly, the solitary wave deforms adiabatically with the production of a trailing shelf. In this paper we re-examine this latter case, and show that the trailing shelf, on a very long time-scale, can lead to the generation of small secondary solitary waves. This result thus provides a connection between the adiabatic deformation regime, and the fission regime.
\end{abstract}

\section{Introduction}

The variable-coefficient Korteweg-de Vries (KdV) equation

$$
u_{s}+\alpha(s) u u_{x}+\lambda(s) u_{x x x}=0 \text {, }
$$

where $\alpha(t), \lambda(t)$ are specified functions of $t$, is well-known as a model equation describing propagation of weakly nonlinear, weakly dispersive waves in inhomogeneous media. For instance, it describes the propagation of shallow-water solitary waves moving over variable depth (Kakutani, 1971, Johnson, 1973), internal solitary waves in regions of variable topography and background stratification (Grimshaw, 1981), inertial solitary waves in rotating fluids (Leibovich and Randall, 1971), and many other situations. Often, in these applications, $s$ is an evolution variable along the path of the wave, and $x$ is a phase variable in terms of which the wave profile is described. Here, however, we shall for convenience refer to $s$ as time, and $x$ as space. Generally, equation (1) is not integrable by the inverse scattering transform method, with some exceptions when the coefficients $\alpha(s)$ and $\gamma(s)$ satisfy some special relations (see Brugarino, 1989, and Dai, 1999.

The variable-coefficient KdV equation (1) has been extensively studied by a combination of asymptotic methods and numerical computations. From these, two contrasting scenarios have emerged. Suppose, for conceptual convenience, that the coefficients $\alpha, \lambda$ are constant 
for $s<0$ and then vary to some different constant values for $s>s_{1}>0$. Then suppose that a solitary wave solution of the constant-coefficient $\mathrm{KdV}$ equation exists for $s<0$ and propagates towards the variable zone. If the temporal scale of the solitary wave is much greater than $s_{1}$, then the wave effectively transits the variable zone unchanged in form, but in the region $s>s_{1}$ it is no longer an exact solution, and instead at $s=s_{1}$ it forms an effective initial condition for another constant-coefficient KdV equation. This, through the inverse scattering transform, then leads to the possibility that it may fission into two or more solitary waves.

On the other hand, if the temporal scale of the solitary wave is much less than $s_{1}$ (that is, much less than the temporal scale on which the coefficients $\alpha$ and $\lambda$ vary), then the wave transforms adiabatically, leaving a trailing shelf behind (see, for instance, Grimshaw, 1979, Knickerbocker and Newell, 1980, Newell, 1985 or Grimshaw and Mitsudera, 1993, and the references therein). This is most easily seen through the following conservation laws for momentum and mass respectively,

$$
\begin{gathered}
\int_{-\infty}^{\infty} \frac{1}{2} u^{2} d x=\text { constant } \\
\int_{-\infty}^{\infty} u d x=\text { constant }
\end{gathered}
$$

A systematic asymptotic expansion shows that the slowly-varying solitary wave deforms in such a way as to conserve its momentum, but having only a single available parameter, cannot then also conserve mass. To remedy this one has to introduce a trailing shelf behind the solitary wave. The typical shelf amplitude is determined by the value of the inhomogeneity gradient, $\epsilon$ say, where $\epsilon \sim \alpha_{s} \sim \lambda_{s}$, while its length is of order $O\left(\epsilon^{-1}\right)$, so that the shelf carries $O(1)$ mass. To leading order this trailing shelf is a linear long wave.

The formation and linear evolution of these trailing shelves has been studied extensively (see the papers already cited and the references therein). In particular, it was noted by Newell (1985, Chapter 3) that when the shelf has a positive polarity, there is the potential for it to be decomposed into a large number of small solitary waves. These secondary solitary waves should become visible for larger times $\left(s \gg \epsilon^{-1}\right)$ when nonlinear effects in the shelf propagation would accumulate, and then compete with dispersive effects. Indeed, various numerical computations for (1) with a more rapid dependence of $\alpha(s)$ and $\lambda(s)$ on $s$ than the slowly-varying theory assumes, show the generation of small solitary waves behind the main solitary wave (see for instance Johnson, 1972, Van Daalen et al, 1997, Pudjaprasetya, 1996 and Pudjaprasetya et al, 1999). Although this generation of secondary solitary waves from the trailing shelf has already been observed and predicted, an explicit analytic description has so far not been available.

In this paper, we re-examine the asymptotic theory for the slowly-varying solitary wave and its accompany trailing shelf. In Section 2 we formulate the problem and then develop the asymptotic theory for the trailing shelf for times of $O\left(\epsilon^{-2}\right)$. On this very long-time scale, nonlinear effects may locally transform the trailing shelf and produce a steepening effect (i.e. incipient shock formation), which in turn then invokes the finer-scale dispersive effects. The outcome is the generation of an undular bore, whose leading waves can be interpreted 
as secondary solitary waves. This process for the formation of an undular bore within the framework of the constant-coefficient $\mathrm{KdV}$ equation has been described by Gurevich and Pitaevskii (1974) and Whitham (1974). The detailed adaptation of that theory to this present circumstance is described in a companion paper by El and Grimshaw (2002). In Section 3 we discuss some explicit applications.

\section{Asymptotic analysis}

We will assume that both coefficients in (1) do not vanish for any $t$, and then without any loss of generality, we can assume that $\alpha>0, \lambda>0$. The case when $\alpha$ passes through zero leads to a breakdown of a solitary wave, and needs a different study from that proposed here (see, for instance, Grimshaw et al 1999, and the references therein. Next, it is convenient to introduce the new variables

$$
t=\int_{0}^{s} \alpha\left(s^{\prime}\right) d s^{\prime}, \quad \text { lam } \hat{b} d a t=\frac{\lambda}{\alpha} .
$$

Then, on omitting the superscript, equation (1) becomes

$$
u_{t}+u u_{x}+\lambda(t) u_{x x x}=0 .
$$

Next, we assume that $\lambda$ is slowly varying, so that

$$
\lambda=\lambda(T), \quad T=\epsilon t, \quad \epsilon \ll 1 .
$$

Then the slowly-varying solitary wave asymptotic expansion is given by

$$
u=u_{0}+\epsilon u_{1}+\ldots,
$$

where

$$
u_{0}=a \operatorname{sech}^{2}\left\{\gamma\left(x-\frac{\phi(T)}{\epsilon}\right)\right\}
$$

and

$$
\frac{d \phi}{d T}=c=\frac{a}{3}=4 \lambda \gamma^{2}
$$

The variation of the amplitude $a$, the inverse-width parameter $\gamma$ and the speed $c$ with the slow time variable $T$ are determined by the momentum conservation law (2), so that,

$$
\int_{-\infty}^{\infty} \frac{1}{2} u_{0}^{2} d x=\text { constant }
$$

Substitution of (8) into (10) readily shows that

$$
\frac{\gamma}{\gamma_{0}}=\left(\frac{\lambda_{0}}{\lambda}\right)^{2 / 3}
$$


where the subscript ' 0 ' indicates quantities evaluated at $T=0$. We also assume, without loss of generality, that $\phi(0)=0$.

From (8), (9) and (11) it follows that The slowly-varying solitary wave $u_{0}$ is now completely determined. However, as is now well-known (see, for instance, Grimshaw and Mitsudera 1993), $u_{0}$ by itself cannot conserve mass. Instead, the conservation of mass is assured by the generation of a trailing shelf $u_{s}$, which typically has an amplitude $O(\epsilon)$ but stretches over a zone of $O\left(\epsilon^{-1}\right)$, and hence has $O(1)$ mass. The law (12) for conservation of mass then shows that

$$
\int_{-\infty}^{\phi / \epsilon} u_{s} d x+\int_{-\infty}^{\infty} u_{0} d x=\text { constant }
$$

The second term on the left-hand side of (12) is readily found to be

$$
\frac{2 a}{\gamma}=24 \lambda \gamma=24 \lambda_{0} \gamma_{0}\left(\frac{\lambda}{\lambda_{0}}\right)^{1 / 3}
$$

on using (8), (9) and (11) in turn. Next, we assume that $u_{s}=\epsilon v(X, T)$, where

$$
X=\epsilon x, \quad T=\epsilon t .
$$

Since $u_{s}$ must satisfy (5) it follows that

$$
v_{T}+\epsilon v v_{X}+\epsilon^{2} \lambda(T) v_{X X X}=0 .
$$

Next, on differentiating (12) with respect to $T$, we find that to leading order in $\epsilon$

$$
v=-\frac{2}{\gamma_{0}} \tilde{\lambda}_{T} \tilde{\lambda}^{-1 / 3} \equiv Q(T), \quad \text { on } X=\phi(T) .
$$

where $\tilde{\lambda}=\lambda / \lambda_{0}$. Note that indeed $v$ is $O(1)$, and so $u_{s}$ is $O(\epsilon)$ as anticipated. Now, (15), (16) together with (9) and (11) present a completely formulated boundary-value problem, and so determine $v$ in the region $X<\phi(T)$ behind the solitary wave.

The usual approach is to take account of only the leading order term in (15), so that $v_{T} \approx 0$, and then

$$
v=v(X), \quad \text { where } \quad v(\phi(T))=Q(T) .
$$

However, the expression (17), being independent of $T$ cannot allow the formation of a secondary solitary wave. Hence, to predict the possible occurrence of secondary solitary waves, it is necessary to retain all the terms in (15).

First, we retain just the nonlinear term, so that equation (15) is reduced to the Hopf equation

$$
v_{T}+\epsilon v v_{X}=0
$$

with the same boundary condition (16). The solution to (18) is readily found using the characteristic equations

$$
\frac{d X}{d T}=\epsilon v, \quad \frac{d v}{d T}=0 .
$$


The solution satisfying the boundary condition (16 is then

$$
v=Q\left(T_{0}\right), \quad X-\phi\left(T_{0}\right)=\epsilon Q\left(T_{0}\right)\left(T-T_{0}\right),
$$

where $T_{0}$ is a parameter along the initial curve $\phi(T)$.

The expression (20) remains valid until neighbouring characteristics intersect and a shock (i.e. a discontinuity in $v$ ) begins to form. This occurs when

$$
c\left(T_{0}\right)-\epsilon Q\left(T_{0}\right)+\epsilon Q_{T}\left(T_{0}\right)\left(T-T_{0}\right)=0 .
$$

Here we have used the result that $d \phi / d T=c$, see (9). Since $\epsilon \ll 1$, it follows that the characteristics will lie in $X<\phi(T)$ and that $c\left(T_{0}\right)-\epsilon Q\left(T_{0}>0\right.$. Hence, from (21) a shock will form in finite time only if $Q_{T}\left(T_{0}\right)<0$ at least for some values of $T_{0}$. Let $T_{b}$ be the minimum value, as $T_{0}$ varies, such that (21) is satisfied. Then a shock forms first at $T_{b}$ and the corresponding value $X_{b}$ is determined from (20). It can be easily seen from (21) that, provided that $Q_{T}\left(T_{0}\right)$ is not zero and is $O(1)$, then $T_{b}$ is $O\left(\epsilon^{-1}\right)$.

However, the full equation (15) cannot support a shock solution, and instead, the shock should be replaced by an undular bore, that is, a modulated periodic wave train which provides a resolution of the shock through the generation of nonlinear small-scale oscillations (see, for instance, Gurevich and Pitaevskii (1974) and Whitham (1974)). The undular bore has solitary waves at its leading edge, and then through a modulated cnoidal-wave regime, gradually degenerates into small-amplitude linear waves at the trailing edge. We interpret the solitary waves at the leading edge of this undular bore as secondary solitary waves generated as the primary solitary wave passes through the variable region.

It follows immediately from (15) that the typical wavelength of these waves is $O\left(\epsilon^{1 / 2}\right)$, the typical period is $O\left(\epsilon^{-1 / 2}\right)$, while a typical amplitude is $O(1)$ in the $X, T, v$ variables; in the original $x, t, u$ variables, the wavelength is $O\left(\epsilon^{-1 / 2}\right)$ (greater than that of the primary solitary wave), the period is $O\left(\epsilon^{-3 / 2}\right)$, and the amplitude is $O(\epsilon)$; the phase speed is $O(\epsilon)$. Hence the temporal scale of the nonlinear waves in the undular bore is much greater than the temporal scale for the variation of $\lambda(T)$ (which is $O(1)$ in the $T$-variable), but in turn much less than the typical formation time $T_{b}$ (which is $O\left(\epsilon^{-1}\right)$ in the $T$-variable). The theory of the undular bore is based on the constant- coefficient $\mathrm{KdV}$ equation, whereas (15) contains the variable coefficient $\lambda(T)$. However, let us now recall, that we can make the plausible assumption that $\lambda(T)$ changes smoothly from a constant value $\lambda_{0}$ for $T<0$ to another constant value $\lambda_{1}$ for $T>T_{1}$. Then, since the undular bore forms in the region where $T \geq T_{b}$ where $T_{b}$ is $O\left(\epsilon^{-1}\right)$, it follows that the governing equation (15) has constant coefficients in the region of interest, and so the semi-classical theory of Gurevich and Pitaevskii (1974) and Whitham (1974) can be directly applied. The detailed description of this study is contained in a companion paper by El and Grimshaw (2002).

The preceding discussion assumes that $\tilde{\lambda}(T)$ is a smooth function, and in particular has a continuous second derivative, so that $Q(T)$ has a continuous first derivative (see (16)). However, in previous studies it has often been supposed that although $\tilde{\lambda}(T)$ is continuous, there may be discontinuities in the derivative $\tilde{\lambda}_{T}$ at $T=0$ and $T=T_{1}$, thus generating jump discontinuities in $Q(T)$. Strictly speaking the preceding asymptotic analysis should not be 
used in the vicinity of $T=0$ and $T=T_{1}$. Nevertheless, it is instructive to assume that the slowly varying solitary wave analysis holds for $0<T<T_{1}$, and to explore the consequences of the predicted jump discontinuities in $Q$ at $T=0$ and $T=T_{1}$. Note that from (16), $Q$ is zero for $T<0$ and $T>T_{1}$. For simplicity, we shall suppose that either $Q(T)>0$, or that $Q(T)<0$ for $0<T<T_{1}$.

Consider first the case when $Q(T)>0$ in $0<T<T_{1}$ (i.e. $\lambda_{T}<0$ ), so that $Q(0+)>0$ and $Q\left(T_{1}-\right)>0$. The positive discontinuity at $T=0$ generates an expansion fan emanating from the point $X=0, T=0$, and given by $v=X / \epsilon T$ in the region $0<X / \epsilon T<Q(0+)$. This expansion fan also solves the full equation (15), and we can infer that no solitary waves are formed from this expansion fan alone. The discontinuities in the derivatives of $v$ at the edges of the expansion fan can be resolved with oscillatory wave trains (Gurevich and Pitaevskii, 1974).

On the other hand, the positive jump discontinuity in $Q$ at $T=T_{1}$ immediately generates a shock; within the framework of the Hopf equation (18) it has an initial amplitude of $Q\left(T_{1}-\right)$ and an initial speed of $\epsilon Q\left(T_{1}-/ 2\right.$. But, within the framework of the full KdV equation (15), the shock is resolved as an undular bore, whose leading edge consists of solitary waves, which are connected through a modulated cnoidal wavetrain to linear sinusoidal waves. After some time $T>T_{i}$ of $O\left(\epsilon^{-1}\right)$, the undular bore and the expansion fan intersect, and there is a complicated interaction process. A detailed description of this process is described by El and Grimshaw (2002) for the special case when $Q\left(T_{0}\right)$ is a positive constant throughout the region $0<T<T_{1}$. It is also shown there that eventually, as $T \rightarrow \infty$, the outcome is a large number of very small solitary waves. In general if $Q\left(T_{0}\right) \geq 0$ for all $T_{0}, 0<T_{0}<T_{1}$, then the only shock formed is that at $T=T_{1}$. But if $Q\left(T_{0}<0\right.$ for some $T_{0}, 0<T_{0}<T_{1}$, then another shock will form at $T=T_{b}$ as described above (see (21)). This shock can also be resolved as an undular bore, and eventually there will arise a more complicated scenario of the interaction of these shocks with each other, and with the expansion fan.

Next, consider the case when $Q(T)<0$ in $0<T<T_{1}$ (i.e. $\lambda_{T}>0$ ), so that $Q(0+)<0$ and $Q\left(T_{1}-\right)<0$. The negative discontinuity at $T=0$ generates a shock, which is resolved as an undular bore, while the negative discontinuity at $T=T_{1}$ generates an expansion fan. The situation is analogous to that in the preceding two paragraphs, and has been described in detail by $\mathrm{El}$ and Grimshaw (2002) for the special case when $Q\left(T_{0}\right)$ is a negative constant throughout the region $0<T<T_{1}$. However, in this case, as $T \rightarrow \infty$, the outcome is radiation of small-amplitude sinusoidal waves.

\section{Applications}

\subsection{Linear growth or decay}

Consider the KdV equation with a linear growth (decay) term,

$$
w_{s}+w w_{x}+w_{x x x}+\sigma(s) w=0,
$$

where $\sigma(s)<(>) 0$ is the growth (decay) coefficient. The transformation $w=u \exp (f(s))$ with $\sigma=-f_{s}$ Converts (5) to the variable-coefficient $\mathrm{KdV}$ equation (1) with $\alpha=\exp (f)$ 
and $\lambda=1$. The further transformation (4) then yields equation (5) with $\lambda(t)=1 / \alpha(s)$, where $d t / d s=\alpha(s)$.

Next suppose that $\sigma(s)=0$ for $s<0, s>s_{1}$, and is a constant, $\sigma(s)=\sigma_{0}$ for $0<s<s_{1}$. It follows that for the transformed equation (5), $\lambda=1$ for $t<0, \lambda=1+\sigma_{0} t$ for $0<t<t_{1}$, and $\lambda=\lambda_{1}=1+\sigma_{0} t_{1}$ for $t>t_{1}$, where $t_{1}=\left(1-\exp \left(-\sigma_{0} s_{1}\right)\right) / \sigma_{0}$. The results of Section 2 now reduce to $\gamma / \gamma_{0}=\left(1+\sigma_{0} t\right)^{-2 / 3}(11)$, and $Q=-\left(\left(2 \sigma_{0} / \gamma_{0}\right)\left(1+\sigma_{0} t\right)^{-1 / 3}(16)\right.$ i for $0<t<t_{1}$. Thus for the growth case $\left(\sigma_{0}<0\right.$, there is an expansion fan generated at $t=0$, and an undular bore generated at $t=t_{1}$. Also, in this case $Q_{t}>0$ for all $t, 0<t<t_{1}$, and so the only undular bore is that formed at $t=0$. Note that here we require that $1+\sigma_{0} t_{1}>0$ to prevent any singularity developing in finite time. For the decay case $\left(\sigma_{0}>0\right)$ the undular bore is formed at $t=0$, with an expansion fan formed at $t=t_{1}$. Again $Q_{t}>0$ and so there is just the one undular bore formed.

\subsection{Water waves over variable depth}

A shallow-water solitary wave propagating over variable depth $h(s)$ is described by the variable coefficient KdV equation (1) (Kakutani, 1971, Johnson, 1973), where

$$
\alpha=\frac{6}{(2 c)^{5 / 2} h^{1 / 2}}, \quad \lambda=\frac{h^{3}}{/} 6 c^{3}, \quad u=c\left(\frac{2 c}{h}\right)^{1 / 2} \zeta .
$$

Here $\zeta$ is the elevation of the wave, and $c=(g h)^{1 / 2}$ is the linear long wave speed. The variable $s$ is distance along the propagation path, and the phase variable $x=\left(\int^{x} d x / c-t\right)$. From the analysis of Section 2, and bearing in mind the transformation (4), we readily deduce from (9) and (11) that the solitary wave amplitude varies as $(\alpha / \lambda)^{1 / 3}$, or as $h^{-3 / 4}$. Then, on using the transformation (23) between $u$ and $\zeta$, we get the well-known result that $\zeta$ varies as $h^{-1}$.

After transforming to (5), we find that $\lambda$ there varies as $h^{-9 / 4}$. Then from (16) we find that $Q$ varies as $-h^{-1 / 2} / h_{T}$. For upslope propagation, where $h$ decreases from a constant value $h_{0}$ at $T=0$ to another constant value $h_{1}$ at $T=T_{1}$, we see that $Q>0$ for $0<T<T_{1}$. Hence in this case we expect to find an expansion fan generated at $T=0$, and an undular bore generated at $T=T_{1}$. Conversely, for downslope propagation $\left(h_{1}>h_{0}, Q<0\right)$, we expect to find an undular bore generated at $T=0$, and an expansion fan generated at $T=T_{1}$. These predictions are qualitatively in agreement with numerical simulations (e.g. Johnson, 1972, Van Daalen et al, 1997, Pudjaprasetya, 1996 and Pudjaprasetya et al, 1999) in that we conclude that secondary solitary waves are formed over the shelf in the case of upslope propagation, whereas for downslope propagation, a radiation field is formed at the onset of the slope.

\section{References}

[1] Brugarino, T. 1989 Painlevé property, auto-Bäcklund transformation, Lax pairs, and reduction to the standard form for the Korteweg-de Vries equation with nonuniformities. J.Math. Phys. $301013-1015$. 
[2] Van Daalen, E.F.G., Van Groesen, E. \& Pudjaprasetya, S.R. 1997 BEM-numerics and KdV-model analysis for solitary wave split up, Computational Mech. 19 179-187.

[3] Dai, H.-H. 1999 Exact solutions of a variable-coefficient KdV equation arising in a shallow water. J.Phys. Soc. Japan 68 1854-1858.

[4] El, G.A. and Grimshaw, R. 2002 Generation of undular bores in the shelves of slowlyvarying solitary waves. Chaos, 12, 1015-1026

[5] Grimshaw, R. 1979 Slowly varying solitary waves. I Korteweg-de Vries equation Proc.Roy.Soc. A 368 359-375.

[6] Grimshaw, R. 1981. Evolution equations for long nonlinear internal waves in stratified shear flows. Stud. Appl. Math., 65, 159-188.

[7] Grimshaw, R. \& Mitsudera, H. 1993 Slowly varying solitary wave solutions of the perturbed Korteweg-de Vries equation revisited. Stud. Appl. Math. 90 75-86.

[8] Grimshaw, R., Pelinovsky, E. \& Talipova, T. (1998). Solitary wave transformation due to a change in polarity. Stud. Appl. Math., 101, 357-388.

[9] Gurevich, A.V. \& Pitaevsky, L.P. 1974 Nonstationary structure of a collisionless shock wave. Sov.Phys.JETP 38 291-297.

[10] Johnson, R. 1972 Some numerical solutions of a variable-coefficient Korteweg-de Vries equation (with application to solitary wave development on a shelf) J.Fluid Mech. $\mathbf{5 4}$ 81-91.

[11] Johnson, R.S. 1973 On an asymptotic solution of the Korteweg-de Vries equation with slowly varying coefficients. J.Fluid Mech. 60 813-824

[12] Kakutani, T. 1971 Effect of uneven bottom on gravity waves, J.Phys. Soc. Japan 30 $272-275$

[13] Knickerbocker, C.J. \& Newell, A.C. 1980 Shelves and the Korteweg-de Vries equation. J.Fluid Mech. 98 803-818.

[14] Leibovich, S. \& Randall, J.D. 1971 Solitary waves in concentrated vortices, J.Fluid Mech. $51625-635$.

[15] Newell, A.C., 1985 Solitons in mathematics and physics. SIAM.

[16] Pudjaprasetya, S.R. 1996 Evolution of Waves above Slightly Varying Bottom: A Variational Approach, Ph.D Thesis, Univ. of Twente, Netherlands.

[17] Pudjaprasetya, S.R., Van Groesen, E. \& Soewono, E. 1999 Splitting of a solitary wave running into shallower water, Wave Motion, 29, 375-389.

[18] Whitham, G.B. 1974 Linear and Nonlinear Waves, Wiley, New York. 